\title{
Effect of hydro mechanical coupling on natural fracture network formation in sedimentary basins
}

\author{
Z. Ouraga ${ }^{1,2} \star$, N. Guy ${ }^{1}$, A. Pouya ${ }^{2}$ \\ ${ }^{1}$ IFP Energies Nouvelles, 1 et 4 avenue du Bois-Préau, 92852, Rueil-Malmaison, France. \\ ${ }^{2}$ Université Paris-Est, Laboratoire Navier (UMR 8205), CNRS, Ecole des Ponts ParisTech, \\ IFSTTAR, Marne la Valle, France.
}

\section{SUMMARY}

In sedimentary basin context, numerous phenomena, depending on the geological time span, can result in natural fracture network formation. In this paper, fracture network and dynamic fracture spacing triggered by significant sedimentation rate is studied considering mode I fracture propagation using a coupled hydro-mechanical numerical methods. The focus is put on synthetic geological structure under a constant sedimentation rate on its top. This model contains vertical fracture network initially closed and homogeneously distributed. The Fractures are modeled with cohesive zone model undergoing damage and the flow is described by poiseuille's law. The effect of the behavior of the rock is studied and the analysis lead to a pattern of fracture network and fracture spacing in the geological layer.

Keywords : Fracture network, hydromechanical behavior, cohesive fracture, damage. 


\section{INTRODUCTION}

Natural fracture network are widely spread in sedimentary basins and can occur in various context involving erosion, tectonic loading or a compaction disequilibrium due to significant sedimentation rate. Depending on the geological time span and the sedimentary basin history, numerous phenomena can lead to a decrease in horizontal effective stress and then gather favorable conditions to fracture onset. In basin modeling, the knowledge of fracturing processes enhances the description of overpressure history, which is a key issue to predict actual overpressures, the fluid content of the underground, the potential locations of hydrocarbon storage and the matrix equivalent permeability. The difficulty in fracture network modeling is to create realistic model due to the lack of sub-surface data (Nelson 2001) and to handle the modeling of the complex physical phenomena involved. Previous papers have studied fracture network within sedimentary rock (Bai and Pollard 2000a; Becker and Gross 1996; Helgeson and Aydin 1991; Priest and Hudson 1976; Rozhko et al. 2007) and fracture initiation and propagation (Simpson et al 2001; Vu et al. 2017). Bai and Pollard (2000a) have shown that in the case of opening mode fracture, the normal stress between two neighboring fractures can change from tensile to compressive depending on the critical ratio of the fracture spacing to layer thickness (approximately equal to 1). Nevertheless, outcrops observation shows ratio of the fracture spacing to layer thickness lower than this critical value (Ladeira and Price 1981; Bai and Pollard 2000b; Narr and Suppe 1991; Gross 1993). Thus, Bai and Pollard (2000a) called to mind other potential mechanisms such as overburden load and/or fluid pressure to explain closely spaced fractures at depth (Narr and Suppe 1991; Rives et al. 1992; Becker and Gross 1996).

In this paper, natural vertical opening-mode fracture network and dynamic fracture spacing induced by significant sedimentation rate are studied using a coupled hydro-mechanical simulator. Assumption of vertical fracture can be considered as a relevant hypothesis in our case of low ratio of horizontal total stress to vertical stress (Pollard and Aydin 1988; Guy et al. 2010). A particular emphasis is put on a synthetic geological structure on which a constant sedimentation rate is imposed on its top. This synthetic geological structure contains vertical fracture network paths initially closed and homogeneously distributed. The Fractures are modeled with cohesive zone model undergoing damage and the flow in the fractures is described by Poiseuilles law. The damage parameter affects both the mechanical and the hydraulic opening of the fracture as discussed by Ouraga et al. (2017). For the numerical simulations, the code Porofis based on finite element modeling (FEM) is used. In the following, the model and 
governing equations are briefly described and then the effect of hydro-mechanical parameters is studied to analyze fracture network and fracture spacing in sedimentary basins.

\section{MODEL AND GOVERNING EQUATIONS}

One of the most important mechanisms involved in natural fracturing in sedimentary basins is the fluid overpressure (Luo and Vasseur 2002; Secor 1965; Mandl 2005). In a sedimentary basin, overpressures are generally due to mechanisms such as mechanical compaction, hydrocarbon maturation, or mineralogical reactions that produce additional pore pressure. Fracture onset occurs when the fluid pressure in a defect reaches the limit tensile strength of the porous media (Ouraga et al. 2017; Fisher et al. 1995). Numerous studies have given mechanical or hydraulic explanation for fracture network spacing (Bai and Pollard 2000a; Gross 1993; Hobbs 1967; Mandl 2005). Nevertheless, in sedimentary basins the coupling between hydraulic and mechanical processes is dominant (Engelder and Fisher 1994; Li et al. 2012). Fracture network is studied from an equivalent model of a synthetic two layer model composed of a caprock formation with a low permeability over a highly permeable reservoir (figure 1). This equivalent model already considered for the modeling of single fracture propagation in sedimentary basins has been widely described in (Ouraga et al. 2017). Thus, by considering that the permeability of the caprock formation is smaller than the permeability of the reservoir, under significant sedimentation rate, the flow from the reservoir to the sealing formation is low enough to represent a negligible part of the amount of fluid in the reservoir. The reservoir can thus be considered as undrained, and the pore pressure in this case reads to (Ouraga et al. 2017):

$$
p(t)=\frac{-b_{r}}{\frac{3\left(1-\nu_{r}\right) K_{r}}{M_{r}\left(1+\nu_{r}\right)}+b_{r}^{2}} \sigma_{v} .
$$

Where $b_{r}, M_{r}, \nu_{r}, K_{r}$ are respectively the Biots coefficient and modulus, Poissons coefficient and bulk modulus of the reservoir. $\sigma_{v}(t)$ is the vertical stress that evolves linearly with time. In the present case to describe the deposit of sediments over time, this vertical stress is imposed on the top of the model and a constant sedimentation rate is assumed. The lower bound of the caprock is loaded with the pore pressure corresponding to the pressure within the reservoir (equation 1). The pressure at the top of the model is taken as a reference pressure and the context is globally oedometric. It means that the vertical displacement of the basement and the horizontal displacement of the two lateral sides of this model are blocked. The principle to initiate fracture is that the low permeability of the caprock prevent overpressure dissipation under significant sedimentation rate (Osborne and Swarbrick 1997). This increase of pore 
pressure by hydro-mechanical coupling leads to an increase of horizontal total stress in the model and with a homogeneous medium does not allow to initiate fractures. Nevertheless, with a perturbation of pore pressure from abnormal conditions such as existing defect associated with an heterogeneity (Figure 1a), fractures can be initiated. For the numerical simulation of fractures onset and their propagation, the caprock has a height of $20 \mathrm{~m}$ and extend over a length of $48 \mathrm{~m}$. Cohesive joints element initially closed are introduced into the mesh of the caprock on predetermined vertical paths, and corresponding to potential fracture propagation paths (figure 1b). It can be considered as a relevant hypothesis in the context of low horizontal total stress to vertical stress ratio. The space between the predetermined vertical paths is $4 \mathrm{~m}$. Each cohesive joint element in the caprock formation is divided into two parts. The first part is the hydraulic defect. The hydraulic defect in this model is initially mechanically closed and represents the hydraulic perturbations resulting from material defects such as roots, shells or bioturbations, which can be inserted in the material during the sedimentation. This defect is around $1 \mathrm{~m}$ length and is very permeable with a dimensionless parameter $\lambda=\frac{C_{f} \mu}{2 \pi k L}>100$ (with $C_{f}$ the fractures conductivity, $k$ the matrix permeability, and $L$ the fractures length). In this framework, with a ratio of fracture conductivity to the matrix permeability $\lambda>100$, the pressure is constant along the defect and equal to the pressure in the reservoir. In the second part of the cohesive zone, the parameter $\lambda$ is closed to 0 , which means that this part is initially inexistent for hydraulic computation. Mechanical and hydraulic parameters are chosen such that the initial fracture has no mechanical influence on numerical simulation until fracture initiation which is associated with the onset of damage in the joint.

The caprock is considered as a porous medium. We assume that the fluids compressibility is very low (usual water properties are considered), the porous medium is isotropic, the skeleton transformations are infinitesimal, and the behavior of the porous material is poroelastic. We also assume that the fluid saturates the pore volume of the media and the flow in the porous media is governed by Darcy's law. As discussed by Coussy (2004) and considering the assumptions above, the governing equation describing the skeleton deformation and the motion of fluid is given by :

$$
\frac{1}{M} \frac{\partial p}{\partial t}=\operatorname{div}\left(\frac{k}{\mu} \nabla p\right)-b \frac{\partial \epsilon_{v}}{\partial t}
$$

where $M$ and $b, \nabla, \epsilon_{v}$ represent respectively the Biot's modulus, the Biot's coefficient, the grandient operator and the volumetric strain given by the trace of the strain tensor. For the mechanical computation the main equations are the equilibrium equation $\operatorname{div}(\underline{\underline{\sigma}})=0$, and the constitutive law of the medium. In this study, by considering the poroelastic behavior, the total stress applied into the medium is: 
(a)

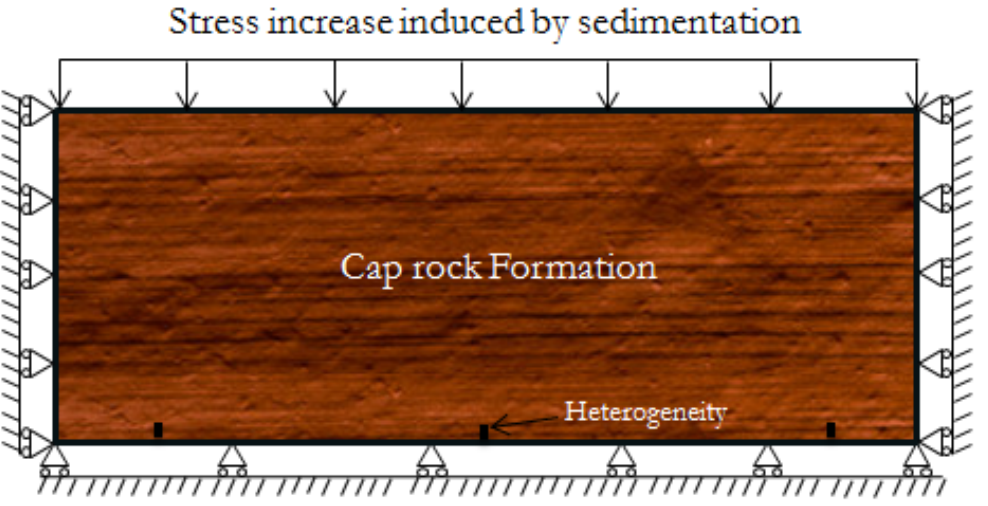

(b)

$p=0: \dot{\sigma}_{v}=\sigma_{a}=C^{t e}$

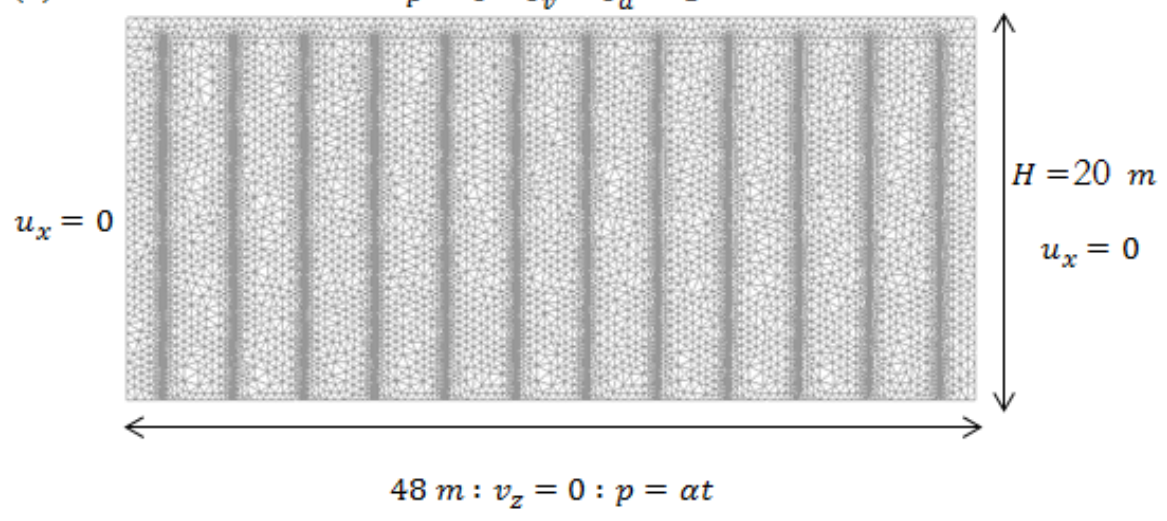

Figure 1. (a) conceptual model and mechanical boundary conditions, (b) finite element method (FEM) numerical simulation model with several vertical predetermined fracture paths, $\alpha=\frac{b}{\frac{3\left(1-\nu_{r}\right) K_{r}}{M\left(1+\nu_{r}\right)}+b^{2}} \sigma_{a}$

$$
\underline{\underline{\sigma}}=\underline{\underline{\underline{\underline{C}}}}: \underline{\underline{\epsilon}}-b p \underline{\underline{I}}
$$

where $\underline{\underline{\underline{\underline{C}}}}$ represents the elasticity tensor, and $\underline{\underline{\epsilon}}$ is the strain tensor. Sign convention of continuum mechanics is used, stress and strain are positive in tension. The flow within the fracture is governed by the Poiseuille's law and the conductivity of the fracture is given by the cubic law. From the mass balance within the fracture, the fluid exchange between the porous medium and the fracture, the hypothesis of continuity of pressure between two fractures surfaces and the Poiseuille's law, the pressure along the fracture surface (Pouya 2012) reads:

$$
\operatorname{div}\left(c_{f} \nabla_{s} p\right)=\frac{e}{K_{f}} \frac{\partial p}{\partial t}+\|v\| \cdot n+\frac{\partial e}{\partial t}
$$

where $e$ is the fractures aperture, $\nabla_{s}$ the tangent gradient in the local tangent plane to the fracture, $C_{f}$ the conductivity in the fracture and $K_{f}$ the fluid compressibility. The velocity $v$ can be discontinuous with the value $v^{+}, v^{-}$on the two sides of the fracture and the operator $\|\cdot\|$ 


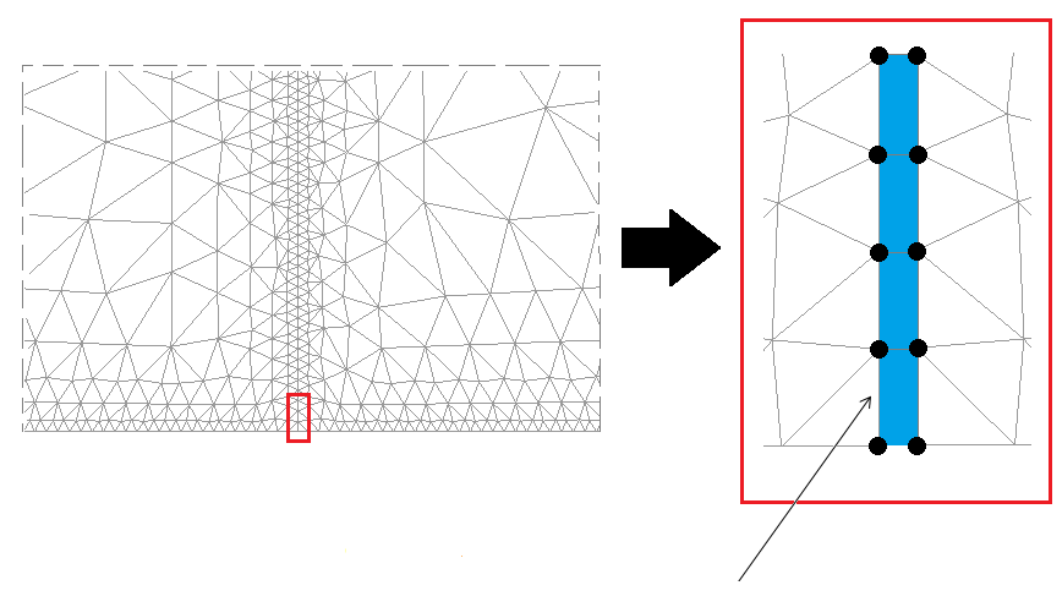

cohesive element with 4 nodes

Figure 2. the mesh around the cohesive zone

represents a jump across the fracture. The damage of cohesive joint is coupled with elasticity using the equation below:

$$
\underline{\sigma_{J}}=(1-D) \underline{\underline{R}} \underline{u}
$$

where $\underline{\sigma_{J}}, D, \underline{\underline{R}}$, are respectively effective stress vector, the damage variable, and the stiffness tensor of the fracture. The vector $\underline{u}$ is the relative displacement of the joint element, and represents the jump of discontinuity between the two side of the fracture. For the mechanical problem the node on the cohesive joint are splitted (figure 2), to allow displacement discontinuities across fractures while for the hydraulic problem it is not necessary because the pressure in our case is the same on the two sides of the fracture. On each side of the fracture, the displacement and the pressure of node are the same that in the matrix. The continuity of the pressure at matrix/fracture interface with the equation (4) ensure the mass balance equation as explained by Pouya (2012). For the numerical computations the coupling between mechanical and hydraulic problems is performed with a sequential solving of the two problems and iteration between them. The sequential solving used here consists in solving the flow problem first through considering constant the total mean stress field. Once the flow problem is computed, the mechanical problem is solved by frozen the pressure in equation (3, $5)$ and the volumetric strain term in equation (2) and the change of the fracture apperture in equation (4) are computed explicitly (Kim et al. 2010; Guy et al. 2012).

The evolution of the fracture is controlled by the failure criterion (figure 3) proposed by Pouya and Bemani (2015) and is defined as followed:

$$
F\left(\tau, \sigma_{n}, D\right)=\tau^{2}-\sigma_{n}^{2} \tan ^{2} \varphi+2 g(D) \sigma_{c} \sigma n-g^{2}(D) C^{2}
$$


where $C$ represents the cohesion on intact cohesive fractures, $\tau, \sigma_{n}, \varphi$ are respectively the shear stress, the normal stress the friction angle and:

$$
\sigma_{c}=\frac{C^{2}+\sigma_{R}^{2} \tan ^{2} \varphi}{2 \sigma_{R}}
$$

with $\sigma_{R}$ the limit tensile strength of the intact joints. This criterion depends on the damage and expressed the decohesion of the cohesive fracture under normal and shear stresses. It describes the transition from an intact cohesive fracture with cohesion and a tensile strength to a completely damage fracture with friction. As seen in figure 3, at the final stage of damage this failure criterion becomes identical to a Mohr Coulomb criterion with only a friction angle. The function $g(D)$ in this failure criterion varies between 0 and 1 , and controls the transition from intact to damage fracture. This function is obtained by using the consistency condition of the failure criterion and it is given by:

$$
g(D)=(1-D)(1-\beta \ln (1-D))
$$

with $D=0$, for an intact rock and $g(D)=1$ and for completely damage fracture $D=1$ and $g(D)=0$.

In this study, the evolution of the damage parameter affects also both the joint stiffness and the fracture aperture (Ouraga et al. 2017). It depends on the relative elastic displacement limit of the cohesive fracture $u_{0}$. Under a displacement $u_{0}$ the damage $D$ remains equal to zero and increases exponentially with the normal joint displacement $u_{n}$ (Pouya and Bemani 2015):

$$
D=1-e^{-\frac{\left(u_{n}-u_{0}\right)}{\beta u_{0}}}, \quad u_{n} \geq u_{0}
$$

where $\beta$ characterizes material ductility and varies in $[0, \infty[$. High $\beta$ represents a ductile material on the contrary $\beta=0$ represents a brittle material. 


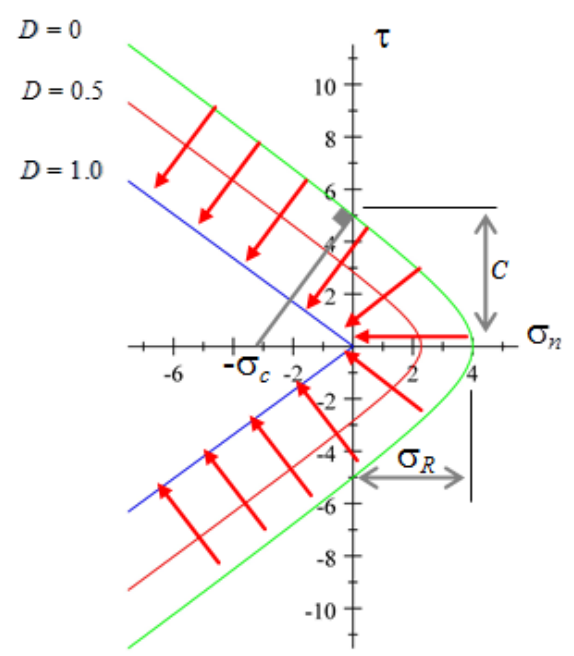

Figure 3. Evolution of the failure criterion from intact cohesive fracture to final damage fracture (Pouya and Bemani 2015)

\section{RESULTS AND DISCUSSION}

The numerical simulations have been carried out considering a ductile and a brittle caprock. The parameters shown in table 1 are used for numerical fracture network initiation and propagation modeling. These parameters are realistic compared to data of poroelastic constant for water-satured rocks (Atkinson and Meredith 1987; Touloukian et al. 1989). In our modeling, the behavior of the media is controlled by the parameter $\beta$ (Pouya 2015) for fixed hydro-mechanical parameters and the results of the simulation are plotted in figure 4 . This figure gives the evolution of pressure fields over time, and the evolution of the geometry of the fracture patterns. In figure 4(a), $\beta=5$ and as discussed by Pouya and Bemani (2015) the cap rock is clearly ductile, while in figure $4(\mathrm{~b}), \beta=0.8$ and the caprock behavior is brittle. Fracture onset in the case of brittle media starts at 1.5 Myrs after the sedimentation and the spacing between fractures is $4 \mathrm{~m}$. This spacing corresponds to the predetermined fracture paths in the media. After the initiation, all fractures have an average length of about $1.5 \mathrm{~m}$ in both cases. This jump corresponds to the mechanical opening of the hydraulic defect $(1 \mathrm{~m})$ and a numerical perturbation in the hydro-mechanical coupling that converges during the computation. Once fractures are initiated the spacing between fractures and their propagation are controlled by the behavior of the caprock. During the propagation, when the fracture length reaches a certain value, some fractures, one of every two fractures stop their propagation and begin to close. Thus the spacing between fractures propagating is multiplied by two. In the ductile caprock as seen in figure 4(a), one of every two fractures seems to close when the fracture length become close to their spacing. In the brittle one, fractures 

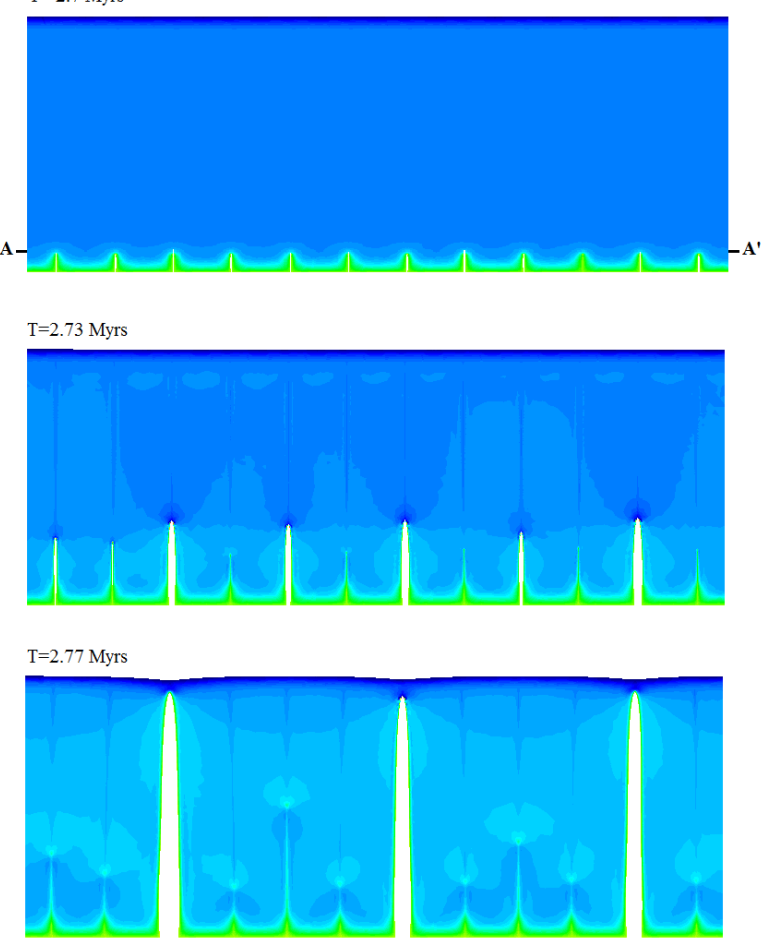

Pore Pressure (MPa) $10.648 \quad 19.166$ (b)

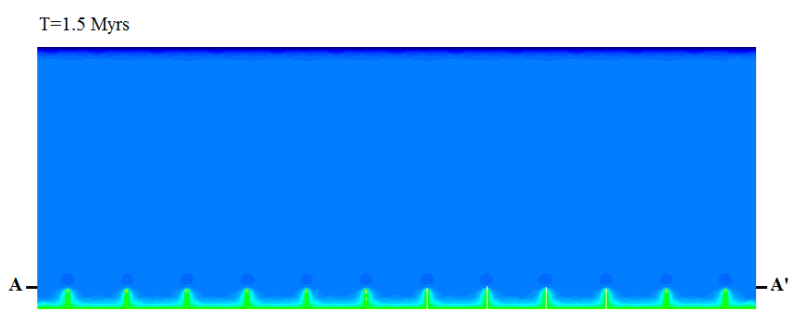

$\mathrm{T}=1.57 \mathrm{Myrs}$

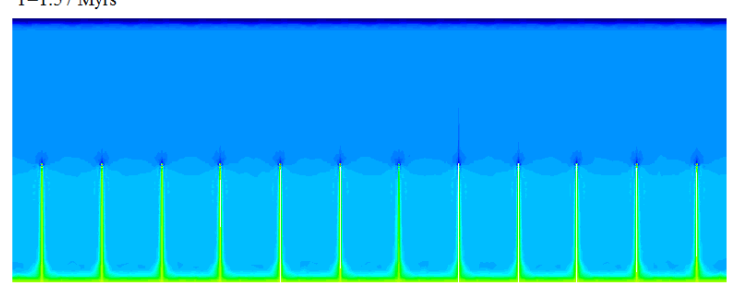

T=1.62 Myrs

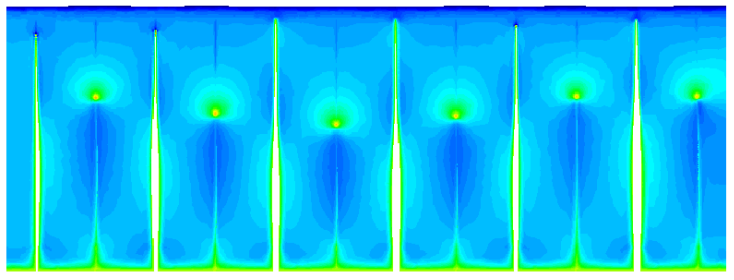

Pore Pressure $(\mathrm{MPa})$ $5.705 \quad 10.351$

Figure 4. fractures propagation and pressure evolution in the case of a ductile caprock (a) and of a brittle caprock (b)

are closed later during their propagation (figure 4(b)). These results allow us to establish a link between the evolution of the spacing and the length of the active fractures depending on the caprock hydro-mechanical properties. The evolution of growing fracture length and the spacing between growing fractures over time are plotted in figure 5 .

In the ductile case (figure 5(a)) the spacing between the fractures evolves with their length. The figure 5(a) justifies the hypothesis made with the analysis of the figure 4 . In fact, according the green and the blue curve, that respectively represent the active fracture length and the average of the spacing between fractures in figure 5(a), one of every two fracture fractures closes when the fracture length become close to their spacing. Graphically, we find this process when these two curves begin to collapse. On the contrary, in the brittle caprock (figure 5(b)) the fractures (one of every two fractures) begin to close when the ratio of fracture length to the spacing becomes clearly smaller than their length (figure 5(b)). As discussed by Bai and Pollard (2000b), these fractures are called closely spaced fractures. In this paper to explain closely spaced fractures, we have focused on the interaction and diffusion between fractures 
(a)

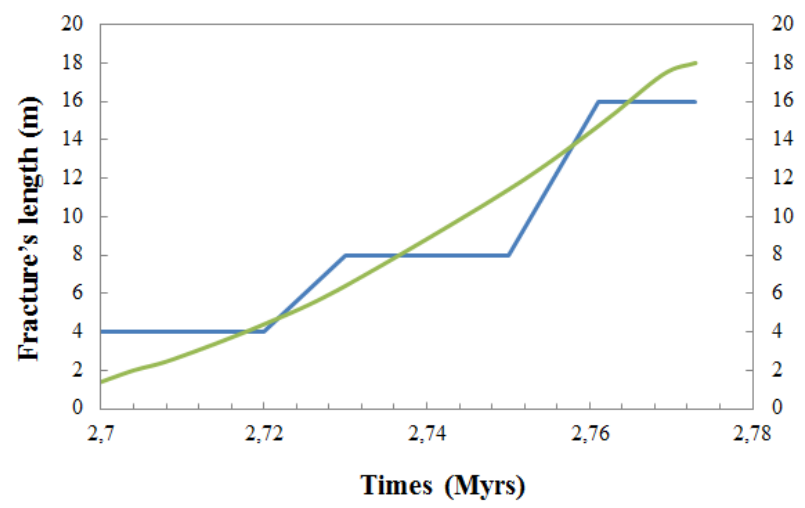

(b)

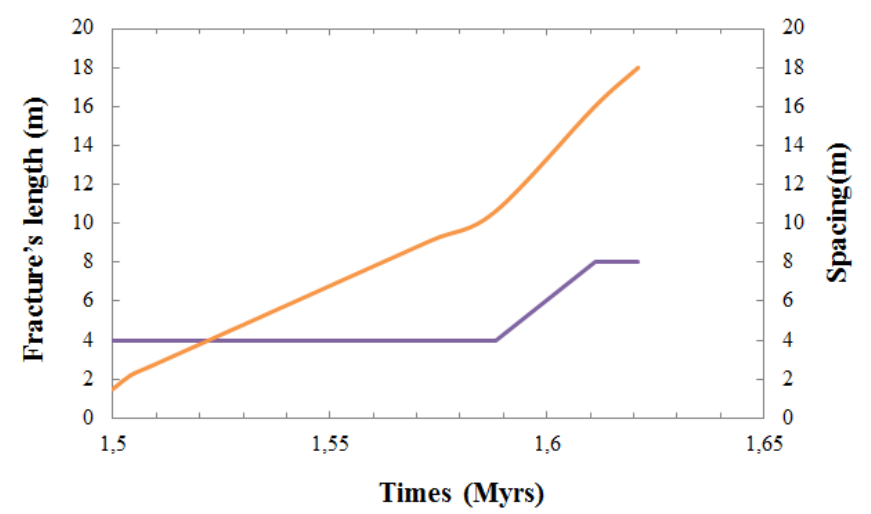

Figure 5. Evolution of fracture's length and of the average spacing between fractures in a ductile caprock (a) and in a brittle caprock(b)

after the initiation (figure 6) and the behavior of the caprock. In the figure 6, the pressure evolution for several periods of time is plotted in a cross section along the line $A A^{\prime}$ (refer to figure 4) of the caprock. This cross section is about at $1.5 \mathrm{~m}$ from the lower bound of the caprock. In this figure each peak of pressure corresponds to the pressure within the fracture in the ductile (figure 6(a)) and the brittle (figure 6(b)) caprock. The blue curve in figure 6 represents the pressure during fracture onset in both cases. In this curve the pressure within all fractures are identical and the effect of diffusion between fractures in the brittle case is not yet established 6(b). The time of fracture onset in this case is very smaller than that in the ductile caprock because, fractures initiate when the limit tensile strength is reached. On the contrary, with $\beta=5$ the softening behavior of the cohesive model leads to a delay in the propagation of fractures compared to the case of a brittle media, when the limit tensile strength is reached. In fact, the stress at the fracture tips in the ductile cap rock must exceed the limit tensile stress to initiate and then to propagate fractures. Therefore, the brittle caprock fracture onset occur in a stress state less compressive than in the ductile case. Thus, the amount of fluid exchange and the flow between fracture and the matrix are small enough during the fracture initiation in the brittle caprock (see blue curves in figure 6) to not allow an increase of the pore pressure far from fractures that could be induced by a flow from fractures to the matrix. In the red curve in figure $6(\mathrm{~b})$ the pressure within all fractures are still identical with a length equal to $10 \mathrm{~m}$ with a spacing of $4 \mathrm{~m}$. As seen in this figure, the pressure between fractures in the brittle caprock does not vary much while in the ductile caprock considering the red and green curves refer to the figure 6(a) the pressure drops in some fractures and halts their propagation by hydromechanical effects. In the brittle caprock, the diffusion effect becomes significant later 

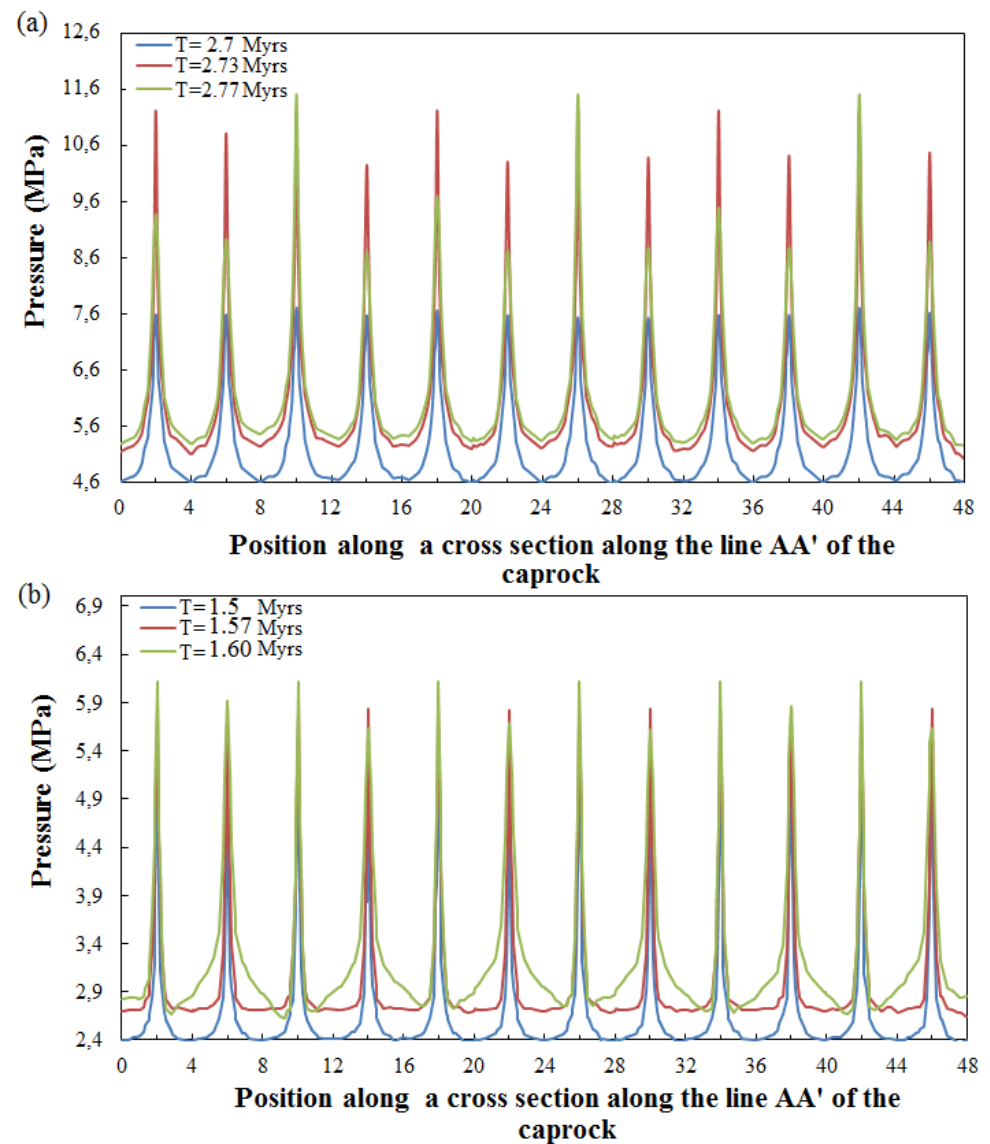

Figure 6. Pressure in a cross section along the line AA' (refer to figure 4) of the caprock for a ductile (a) and brittle (b) behavior

at around 0.1 Myrs after fracture initiation (figure 6(b), 4(b)), and fractures have a length of $16 \mathrm{~m}$. Therefore, the diffusion drops the pressure within some fractures. They begin to feel the influence of the neighboring fractures and a pressure gradient is created within some fractures. Therefore, with the hydro-mechanical coupling and the compressive stress state between the

Table 1. Input data for simulation

\begin{tabular}{ccccccc}
\hline Property & $\mathrm{E}(\mathrm{GPa})$ & $\nu(-)$ & $\mathrm{k}\left(\mathrm{m}^{2}\right)$ & $\beta$ & \\
\hline Caprock & 10 & 0.23 & $10^{-21} m^{2}$ & $0.8 / 5$ & \\
Reservoir & 1 & 0.2 & - & - & \\
\hline Overburden & $200 \mathrm{~m} / \mathrm{Myrs}$ & & & & & \\
\hline Fracture & $\mathrm{R}_{t t}(G P a / m)$ & $\mathrm{R}_{t t}(G P a / m)$ & $\mathrm{R}_{n t}=R_{t n}(G P a / m)$ & $\sigma_{R}(M P a)$ & $\mathrm{C}(\mathrm{MPa})$ & $\varphi(0)$ \\
& 50 & 50 & 0 & 1 & 0.5 & 25 \\
\hline
\end{tabular}


fractures, the propagation criterion is no longer respected and the lips of fractures begin to close (one of every two fractures), the spacing between fracture increases and we find the same interaction between fractures as in the ductile case.

Hence, according our results by considering a very low permeability caprock and a significant sedimentation rate, in the brittle case, it is more likely to have closely spacing fractures than in ductile one due to the effects of hydro-mechanical coupling. These results can be used to explain the formation of fracture corridors, and some patterns of fracture network in the rock under a significant sedimentation rate.

\section{CONCLUSION}

In this paper, we have studied the effect of hydro-mechanical coupling and the mechanical properties of the caprock on the pattern of fracture network and their spacing under significant sedimentation rate. In a ductile caprock the spacing between fracture evolves and can be compared with its length of propagation. One of every two fractures closes when the length of the fracture becomes larger than their spacing. In fact, the influence distance of a fracture on the neighboring fractures is roughly equivalent to its length in this case. During fractures propagation, the diffusion between them and the hydro-mechanical coupling affects the network evolution. In the brittle caprock case some fractures start to close, only when the diffusion between fracture begin to establish. Therefore, closely spaced fracture network can be formed when the fracture length is larger than the spacing between fractures before the diffusion process between fractures becomes significant. This result can explain in-situ observation of closely spaced fractures (Bai and Pollard 2000b; Ladeira and Price 1981) and of some patterns of fracture network under significant sedimentation rate. Finally, our modeling method and our cohesive fracture model can be considered as relevant to compute natural fracture network under sedimentation in a realistic context in single-phase fluid flow. The case of double-phase fluid flow could be integrated in an extended version of this model.

\section{ACKNOWLEDGMENTS}

This work was funded by IFP Energies Nouvelles research program. The data for this paper are available by contacting the corresponding authors at zady.ouraga@ifpen.fr 


\section{REFERENCES}

Atkinson, B. K., and P. G. Meredith (1987), Experimental fracture mechanics data for rocks and mineral, Fracture Mechanics of Rock, San Diego, Calif, 477-525.

Bai, T., and D. D. Pollard (2000a), Fracture spacing in layered rocks: a new explanation based on the stress transition, Journal of Structural Geology, 22, 1409-1425.

Bai, T., and D. D. Pollard (2000b), Closely spaced fractures in layered rocks: initiation mechanism and propagation kinematics, Journal of Structural Geology, 22, 1409-1425.

Becker, A., and M. R. Gross (1996), Mechanism for joint saturation in mechanically layered rocks: an example from southern israel, Tectonophysics, 257, 223-237.

Coussy, O. (2004), Poromechanics, John Wiley \& Son Ltd.

Engelder, T., and M. P. Fisher (1994), Influence of poroelastic behavior on the magnitude of minimum horizontal stress, sh, in ooverpressure parts of sedimentary basins, Geology, 22, 946-952.

Fisher, M. P., M. R. Gross, T. Engelder, and T. Greenfield (1995), Finite-element analysis of the stress distribution around a pressurized crack in a layered elastic medium: implication for the spacing of fluid driven joint in bedded sedimentary rock, Tectonophysics, 247, 49-64.

Gross, M. R. (1993), The origin and spacing of cross joint: example from monterey formation, santa barbara coastline, california, Journal of Structural Geology, 15, 737-751.

Guy, N., D. Seyedi, and F. Hild (2010), Hydro-mechanical modmodel of geological co2 storage and the study of possible caprock fracture mechanisms, Georisk, 4(3), 110-117.

Guy, N., G. Enchery, and R. G (2012), Numerical modeling of thermal eor: comprehensive coupling of an amr-based model of thermal fluid flow and gepmechanics, Oil, 67(6), 1019-1027.

Helgeson, D. E., and A. Aydin (1991), Characteristics of joint propagation across layer interface in sedimentary rocks, Journal of Structural Geology, 13(8), 897-911.

Hobbs, D. W. (1967), The formation of tension jjoint in sedimentary rocks: an explanation, Geol. Mag., 104, 550-556.

Kim, J., H. Tchelepi, and R. Juanes (2010), Stability accuracy and efficiency of sequential methods for coupled flow and geomechanics, in SPE Reservoir simulation symposium. The Woodlands, Texas.

Ladeira, F. L., and N. J. Price (1981), Relationship between fracture spacing and bed thickness., Journal of Structural Geology, 3, 179-183.

Li, C. L., C. A. Tang, and S. Y. Wang (2012), A numerical investigation of fracture infilling and spacing in layered rocks subjected to hydro-mechanical loading, Rock Mech Rock Eng, 45, 753-765. Luo, X., and G. Vasseur (2002), Natural hydraulic cracking: Numerical model and sensitivity study, Earth and Planetary Science Letters, 201, 431-446.

Mandl, G. (2005), Rocks joint: The mechanical genesis, Springer, Heidelberg, pp. 27-48.

Narr, W., and J. Suppe (1991), Joint spacing in sedimentary rocks, Journal of Structural Geology, 13, 1037-1048.

Nelson, R. A. (2001), Geological analysis of naturally fractured reservoir, second edition. Gulf Profes- 
sional Publishing, Houston.

Osborne, M., and R. Swarbrick (1997), Mechanisms for generating overpressure in sedimentary basins, AAP, 81, 1023-1041.

Ouraga, Z., N. Guy, and A. Pouya (2017), Modeling of natural fracture initiation and propagation in basin sedimentation context, Journal of Geophysical Research: Solid Earth, 122, 247-261.

Pollard, D. D., and A. Aydin (1988), Progress in understanding joint over the past century, Geological Society of America Bulletin, 100, 1181-1204.

Pouya, A. (2012), Three-dimensional flow in fractured porous media: A potential solution based on singular integral equations, Advances in Water Ressources, 35, 30-40.

Pouya, A. (2015), A finite element method for modeling coupled flow and deformation in porous fractured media, International Journal for Numerical and Analytical Methods in Geomechanic, 39, $1836-1852$.

Pouya, A., and Y. Bemani (2015), A damage-plasticity model for cohesive fractures, International Journal for Rock Mechanics 83 Minning Sciences, 73, 194-202.

Pouya, A., and S. Ghabezloo (2010), Flow around a crack in a porous matrix and related problems, Transport in Porous Media, 84, 511-532.

Priest, S., and J. A. Hudson (1976), Discontinuity spacing in rocks, International Journal of Rock Mechanics \&3 Mining Sciences, 13, 135-148.

Rives, T., M. Razack, J. P. Petti, and K. Rawnsley (1992), Joint spacing: analogue and numerical simulations, Journal of Structural Geology, 14, 925-937.

Rozhko, A., Y. Podladchikov, and F. Renard (2007), Failure patterns caused by localized rise in pore-fluid overpressure and effective strength of rocks, Geophysical Research Letters, 34, L22,304.

Secor, D. (1965), Role of fluid pressure in jointing, American Journal of Science, 263, 633-646.

Simpson, G., Guguen, Y., Schneider, F. (2001), Permeability enhancement due to microcrack dilantacy in the damage regime, Journal of Geophysical Research: Solid Earth, 106, 3999-4016.

Touloukian, Y. S., W. R. Judd, and R. F. Roy (1989), Physical properties of rocks and minerals, Hemisphere, Bristol.

M. N. Vu, S. T. Nguyen, Q. D. To and N. H. Dao (2017), Theoretical predicting of permeability evolution in damaged rock under compressive stress, 209 (2), 1352-1361.

Zimmerman, R. W., and G. S. Bodvarsson (1996), Hydraulic conductivity of rock fractures, Transport in Porous Media, 23, 1-30.

Zhou, C., Sharma, R., and Y.F., C. (2008). Flow-stress coupled permeability tensor for fractured rock masses. International Journal of Numerical and Analytical Methods in Geomechanics, 32:1289-1309. 